\title{
Les canaux potassiques sensibles à l'ATP ou les suites imprévues de l'étude des sulfamides hypoglycémiants
}

Les sulfamides hypoglycémiants sont de " vieux " médicaments du diabète non-insulinodépendant dont le mécanisme d'action était jusqu'ici inconnu. Ils figurent cependant depuis peu parmi les outils essentiels permettant d'analyser la fonction, la régulation et les implications physiopathologiques d'une classe toute neuve de canaux ioniques, les canaux potassiques sensibles aux variations d'ATP intracellulaire. Ces canaux jouent un rôle-clé dans la sécrétion d'insuline par le pancréas. Ils sont aussi présents, ayant une fonction physiologique importante, dans les cellules cardiaques, le muscle lisse et le système nerveux central. Ils sont en relation étroite avec une variété de maladies qui vont du diabète aux désordres liés à l'ischémie cérébrale, en passant par l'hypertension et les arythmies cardiaques.

\section{Michel Lazdunski}

\section{ADRESSE}

M. Lazdunski : professeur de biochimie à l'université de Nice, directeur du laboratoire de phar macologie moléculaire et cellulaire du Cnrs. 660 , route des Lucioles, Sophia Antipolis, 06560 Valbonne, France.

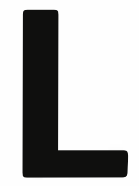

es sulfamides hypoglycémiants sont des sulf onylurées. Ils constituent une grande classe de médicaments utilisés depuis plusieurs décennies [1] pour le traitement du diabète de type II (non insulinodépendant). On sait depuis longtemps que cette classe de molécules a la propriété de provoquer la sécrétion d'insuline par les îlots de Langherans. On ignorait jusqu'à un passé proche le mécanisme moléculaire d'action de ces médicaments antidiabétiques. Il est démontré aujourd'hui [2] que la cible de cette grande classe médicamenteuse est un canal ionique qui joue un rôle central dans le fonctionnement de la cellule $\beta$ pancréatique, le canal potassium sensible à l'ATP.
Les canaux potassium sont présents dans toutes les cellules ou presque. Ils assurent la polarisation de la membrane plasmique. L'ouverture d'un canal $\mathrm{K}^{+}$provoque la sortie de $\mathrm{K}^{+}$, de l'intérieur vers l'extérieur de la cellule (la concentration de $\mathrm{K}^{+}$ intracellulaire est très supérieure à la concentration extracellulaire). Cette ouverture peut être commandée par la variation d'un potentiel de membrane ; il s'agit alors de la classe des canaux $\mathrm{K}^{+}$dits voltage-dépendants qui jouent un rôle central dans toutes les phases de repolarisation des potentiels d'action des cellules excitables. Elle peut aussi être commandée par des variations de concentrations intracellulaires de "messagers" importants tels l'ion $\mathrm{Ca}^{2+}$ (il s'agit alors de canaux $\mathrm{K}^{+}$dits $\mathrm{Ca}^{2+}$. 


\section{RÉFÉRENCES}

1. Loubatières A. Effects of sulfonylureas on the pancreas. In : The Diabetic Pancreas. 1977, 489-515.

2. Schmid-Antomarchi H, De Weille J, Fosset M, Lazdunski M. The receptor for antidiabetic sulfonylureas controls the activity of the ATP-modulated $\mathrm{K}^{+}$channel in insulin-secreting cells. J Biol Chem 1987 ; 262: 15840-4.

3. Petersen $\mathrm{OH}$, Findlay I. Electrophysiology of the pancreas. Physiol Rev 1987 ; 67 : 1054-116.

4. Ashcroft FM. Adenosine 5'-triphosphatesensitive potassium channels. Ann Rev Neurosi $1988 ; 11: 97-118$.

5. Bernardi H, Fosset M, Lazdunski M. Characterization, purification and affinity labelling of the brain $\left[{ }_{3} \mathrm{H}\right]$ glibenclamide binding protein, a putative neuronal ATP. regulated $\mathrm{K}^{+}$channel. Proc Natl Acad Sci USA 198885 : 9816-20.

6. De Weille JR, Schmid-Antomarchi H, Fosset M, Lazdunski M. Regulation of ATP-sensitive $\mathrm{K}^{+}$channels in insulinoma cells. Activation by somatostatin and kinase $\mathrm{C}$ and the role of cAMP. Proc Natl Acad Sci USA 1989 ; 86 : 2971-5.

7. Tatemoto K, Rökaeus AA, Jörnvall $H$, McDonald TJ, Mutt V. Galanin, a novel biologically active peptide from porcine intestine. FEBS Lett 1983 ; 164 : 124-8.

8. De Weille JR, Schmid-Antomarchi H, Fosset M, Lazdunski M. ATP-sensitive $\mathrm{K}^{+}$channels that are blocked by hypoglycemia-inducing sulfonylureas in insulin-secreting cells are activated by galanin, a hyperglycemia-inducing hormone. Proc Natl Acad Sci USA $1988 ; 85: 1312-6$.

9. Quast U, Cook NS. Moving together : $\mathrm{K}^{+}$channel openers and ATP-sensitive $\mathrm{K}^{+}$channels. TIPS $1989 ; 10: 431-5$.

10. Noma A. ATP-regulated $\mathrm{K}^{+}$channels in cardiac muscle. Nature $1983 ; 305: 147-8$.

11. Trube G, Hescheler J. Inwardrectifying channels in isolated patches of the heart cell membranes: ATP-dependence and comparison with cell-attached patches.

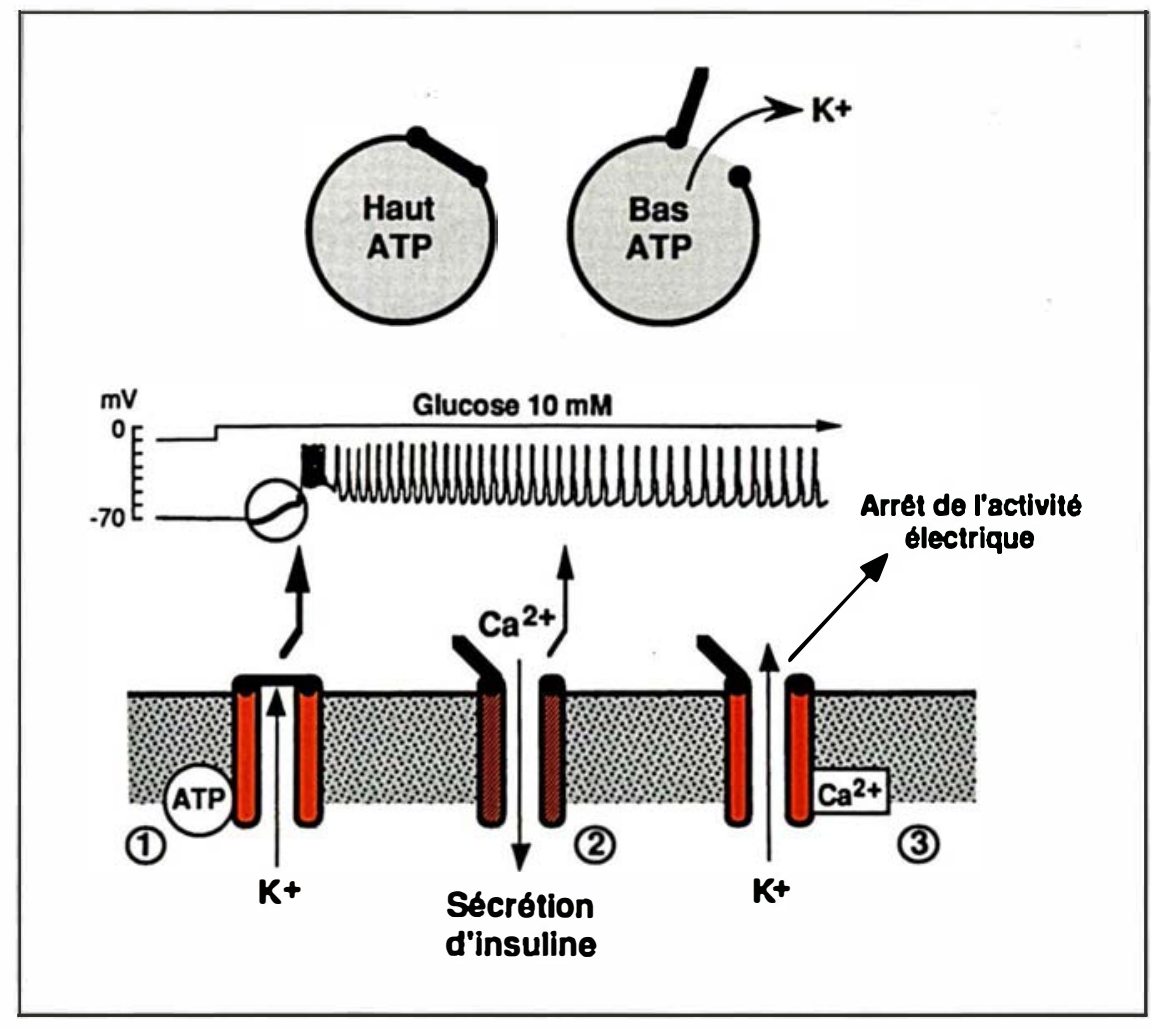

Figure 1. Comment le glucose induit-il l'activité électrique de la cellule $\beta$ pancréatique? Fonctionnement des canaux $K_{A T P}$ ouverts quand la concentration d'ATP est faible, fermés à haute concentration d'ATP (haut). Activité électrique d'une cellule $\beta$ pancréatique en présence de glucose (bas). La dépolarisation initiale est due à la fermeture des canaux $K_{\text {ATP }}$ (1). Elle induit une activité électrique répétitive due aux canaux $\mathrm{Ca}^{2+}$ (2) qui produira l'entrée de $\mathrm{Ca}^{2+}$ et la sécrétion d'insuline. L'arrêt ultérieur de cette activité Inon représentée) (3) est dû à la mise en marche des canaux $\mathrm{K}^{+}$, sensibles au $\mathrm{Ca}^{2+}$ intracellulaire.

dépendants) ou l'ATP cytoplasmique (canaux $\mathrm{K}^{+}$sensibles à l'ATP).

Le canal $\mathrm{K}^{+}$sensible aux variations d'ATP intracellulaire (canal $\mathrm{K}_{\mathrm{ATP}}$ ) assure une relation essentielle entre le niveau bioénergétique de la cellule (ATP) dans laquelle il est présent et les caractéristiques d'excitabilité électrique de la membrane plasmique. $\mathrm{Ce}$ canal est de découverte récente [3-4]. Il se ferme lorsque la concentration intracellulaire d'ATP $\left([\mathrm{ATP}]_{\text {in }}\right)$ augmente, il s'ouvre lorsque la concentration cytoplasmique d'ATP diminue.

L'insuline contrôle le niveau plasmatique de glucose et à l'inverse le niveau de glucose sanguin contrôle l'intensité de la sécrétion d'insuline par la cellule $\beta$ pancréatique. En l'absence d'exposition à une perfu- sion de glucose, la cellule $\beta$ est bien polarisée $(\approx-70$ à $-60 \mathrm{mV})$, elle se comporte comme une cellule non excitable (elle n'engendre pas de potentiels d'action), elle ne sécrète pas (ou peu) d'insuline. Cette situation (bonne polarisation, pas d'activité électrique) est due au fait qu'un certain nombre des canaux $\mathrm{K}^{+}$sont alors ouverts, parmi lesquels les canaux $\mathrm{K}_{\mathrm{ATP}}$. Dès que la cellule $\beta$ est exposée à une perfusion de glucose, elle se dépolarise et cette dépolarisation entraîne l'apparition d'une activité électrique spontanée avec des potentiels d'action répétitifs ; la cellule $\beta$ est devenue excitable. La longueur des trains de potentiels d'action va dépendre en partie de la concentration de glucose utilisée. Après un intervalle de temps qui 


\section{$\underset{100 \mathrm{~ms}}{2} \mathrm{pA}$}

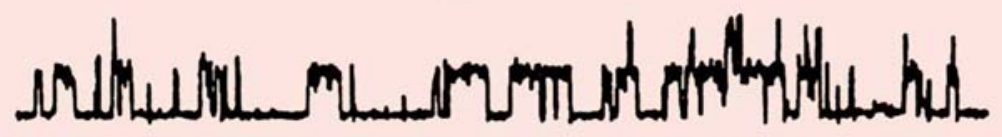

Faible concentration d'ATP cytoplasmique $(1 \mu \mathrm{M})$

ATP cytoplasmique élevé (3-4 mM)

ATP cytoplasmique bas,

application extracellulaire de glibenclamide

Figure 2. Expression du canal $\boldsymbol{K}_{\text {ATP }}$ dans des cellules d'insulinome mesurée par la technique de patch-clamp. Le canal s'exprime quand la concentration d'ATP cytoplasmique est réduite. II est inhibé à plus haute concentration d'ATP. II est aussi inhibé par un sulfonylurée comme le glibenclamide.

peut atteindre des minutes, la cellule $\beta$ redeviendra silencieuse puis reprendra plus tard son activité électrique spontanée si la perfusion de glucose persiste. C'est l'activité électrique spontanée provoquée par la perfusion de glucose qui entraîne la sécrétion d'insuline.

Le mécanisme moléculaire de ce processus électrogène est désormais bien compris. La perfusion de glucose va activer les mécanismes intracellulaires de production d'ATP et cette augmentation d'[ATP $]_{\text {in }}$ va provoquer la fermeture des canaux $\mathrm{K}_{\mathrm{ATP}}$. C'est là l'étape clé de l'ensemble du processus, car cette fermeture de canaux va provoquer la dépolarisation qui entraînera la succession de toutes les autres étapes conduisant à la sécrétion de l'hormone. Cette dépolarisation va en effet conduire le potentiel de membrane au seuil d'activation des canaux calcium $\left(\mathrm{Ca}^{2+}\right)$ voltage-dépendants. L'activation de ces canaux provoque la production de potentiels d'action répétitifs (figure 1). L'ouverture de ces canaux pendant les potentiels d'action permet l'entrée de $\mathrm{Ca}^{2+}$ qui provoquera l'augmentation du $\mathrm{Ca}^{2+}$ intracellulaire nécessaire à la $\mathrm{m} / \mathrm{s} n^{\circ} 3$ vol. 6 , mars 90 supérieures [2]. Les inhibiteurs les plus puissants sont aussi les antidiabétiques les plus puissants [2] (agissent à des doses particulièrement faibles). En bloquant les canaux $\mathrm{K}_{\mathrm{ATP}}$ dépendants, les sulfonylurées produisent les mêmes effets que le glucose (qui inhibe indirectement l'expression de ces canaux en produisant une augmentation d' $\left.[\mathrm{ATP}]_{\text {in }}\right)$.

Tout ce qui est connu aujourd'hui des canaux ioniques au niveau moléculaire l'est grâce à la pharmacologie. Les sulfonylurées les plus puissants ont pu être tritiés, leurs récepteurs identifiés [2] et de ce fait mieux connus au plan moléculaire, y compris pour ce qui concerne leur structure [5].

\section{Les canaux $K_{\text {ATp, }}$ cibles des sulfonylurées antidiabétiques, peuvent s'ouvrir sans qu'il soit nécessaire de faire varier la concentration d'ATP intracellu- laire}

$\mathrm{Si}$ la sécrétion d'insuline par les cellules $\beta$ du pancréas est dépendante du niveau de concentration extracellulaire de glucose, elle est aussi modulée par toute une série d'hormones. La plus connue d'entre elles est la somatostatine. La somatostatine inhibe la sécrétion d'insuline par la cellule $\beta$. Cette inhibition de sécrétion est liée à l'action de l'hormone sur l'expression des canaux $\mathrm{K}_{\mathrm{ATP}}[6]$ (figure 3, p. 282). L'hormone se fixe à son récepteur spécifique, cette fixation amène l'activation d'une protéine $\mathrm{G}$ (protéine qui fixe le GTP) qui provoque elle-même l'activation des canaux $\mathrm{K}_{\mathrm{ATP}}$. Les canaux $\mathrm{K}_{\mathrm{ATP}}$ ainsi activés demeurent blocables par les sulfonylurées. L'activation des canaux $\mathrm{K}_{\mathrm{ATP}}$ par la somatostatine provoquera une hyperpolarisation qui réduira la probabilité d'ouverture des canaux $\mathrm{Ca}^{2+}$, voie d'entrée majeure $\mathrm{du} \mathrm{Ca}^{2}+$ nécessaire à la sécrétion d'insuline, et par conséquent la libération de l'hormone.

Une autre hormone de découverte plus récente [7], la galanine qui, elle aussi, inhibe la sécrétion d'insuline et est dotée d'un eff et hyperglycémiant, fonctionne par le même mécanisme que celui qui a été précédemment décrit pour la somatostatine qui comporte la fixation de l'hormone à son récepteur, l'activation d'une protéine 
$\mathrm{G}$, l'activation des canaux $\mathrm{K}_{\mathrm{ATP}}$. $\mathrm{La}$ protéine $G$ qui couple ces récepteurs d'hormone à l'activation du canal $\mathrm{K}_{\mathrm{ATP}}$ est sensible à la toxine pertussique. Cette toxine inhibe donc l'effet activateur des hormones sur l'activation des canaux $\mathrm{K}_{\mathrm{ATP}}$. La figure 4 montre l'effet de la galanine sur l'activité électrique d'une cellule $\beta$ pancréatique.

Le canal $\mathrm{K}_{\mathrm{ATP}}$ est donc la cible : (1) des sulfonylurées antidiabétiques ; (2) des hormones qui inhibent la sécrétion d'insuline ; (3) du glucose qui stimule la sécrétion d'insuline. Il ne serait pas autrement étonnant qu'une anomalie de structure ou de régulation de ce canal soit à l'origine de l'état diabétique.

Les canaux $K_{\text {ATP }}$ dans le muscle lisse, les ouvreurs de canaux $\mathrm{K}^{+}$, des médicaments nouveaux potentiels pour l'hypertension, l'asthme, l'incontinence urinaire, etc.

Une classe nouvelle de médicaments potentiels vient de naître, celle des ouvreurs de canaux $\mathrm{K}^{+}$(figure 5). Ces molécules - le monoxidil sulfate, le cromakalim, le nicorandil, le pinacidil, le RP 49356 - ont la propriété d'induire la relaxation des muscles lisses [9]. L'ouverture des canaux $\mathrm{K}^{+}$polarise la cellule musculaire lisse, éloigne le potentiel de membrane du potentiel seuil d'activation des canaux $\mathrm{Ca}^{2+}$ (de type $\mathrm{L}$ ), cibles des anticalciques et système d'entrée majeure de $\mathrm{Ca}^{2+}$ dont l'augmentation de concentration cytoplasmique est essentielle pour l'induction de la contraction. Cette hyperpolarisation induite par les ouvreurs de canaux $\mathrm{K}^{+}$diminuera la probabilité d'ouverture des canaux $\mathrm{Ca}^{2+}$ et par conséquent la tendance à contracter.

Les canaux $\mathrm{K}^{+}$ouverts par les ouvreurs (cromakalim, pinacidil, RP 49356) sont bloqués par les sulfonylurées antidiabétiques. Les ouvreurs potassiques sont vasorelaxants, les sulfonylurées éliminent cette vasorelaxation. Les canaux cibles des ouvreurs sont des canaux $\mathrm{K}_{\text {ATP }}$ [9], mais les ouvreurs manifestent leur action sans modifier la concentration d'ATP intracellulaire. La sensibilité des canaux $\mathrm{K}_{\text {ATP }}$ du muscle lisse aux sulfonylurées est bien inférieure à celle observée dans la cellule $\beta$ ( $\approx 1000$ fois). Il est donc probable que les canaux $\mathrm{K}_{\mathrm{ATP}} \mathrm{du}$ muscle lisse soient le produit de gène(s) différent(s) de celui qui est exprimé dans la cellule $\beta$. Inversement d'ailleurs, les canaux $\mathrm{K}_{\mathrm{ATP}} \mathrm{de}$ la cellule $\beta$ pancréatique sont beaucoup moins sensibles aux ouvreurs de canaux $\mathrm{K}^{+}$que la cellule musculaire lisse.

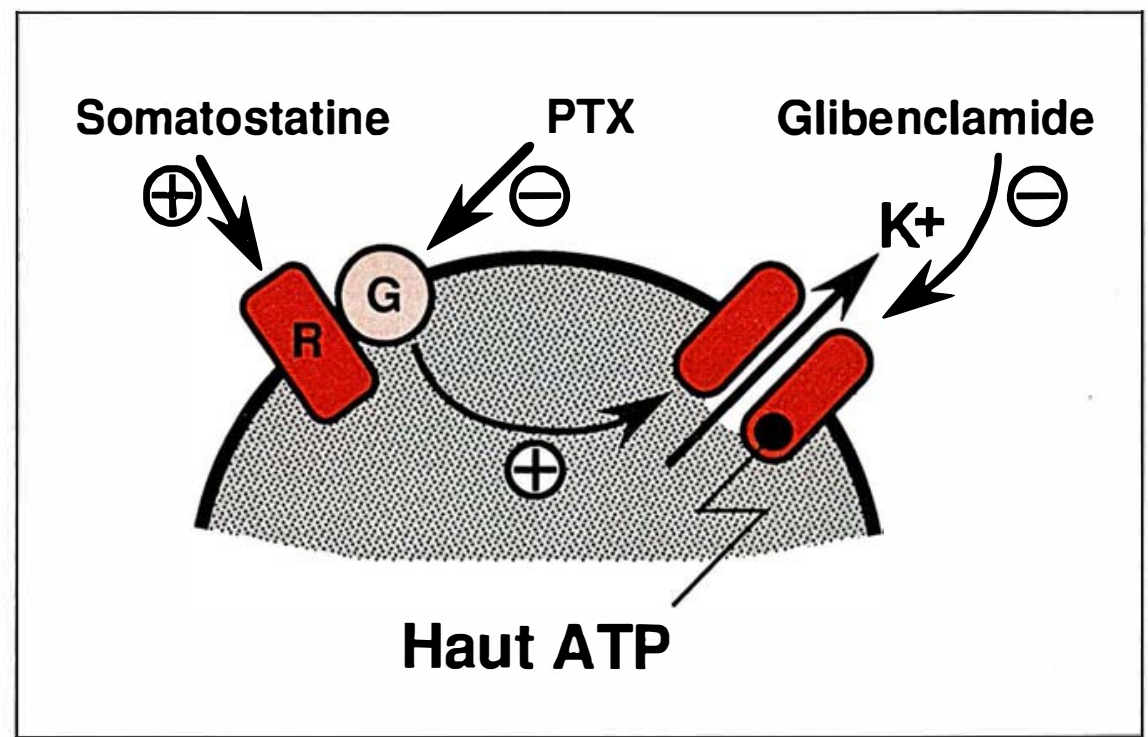

Figure 3. Mécanisme d'action de la somatostatine. La somatostatine se fixe à son récepteur $R$ qui s'associe à une protéine liant le GTP (protéine G) et qui l'active. La protéine $G$ activée entraîne l'activation du canal $K^{+}$sans modification d'ATP intracellulaire. Le canal $\mathrm{K}^{+}$activé par le somatostatine est bloqué par le glibenclamide. L'effet activateur de la protéine $G$ est supprimé par la toxine pertussique (PTX).

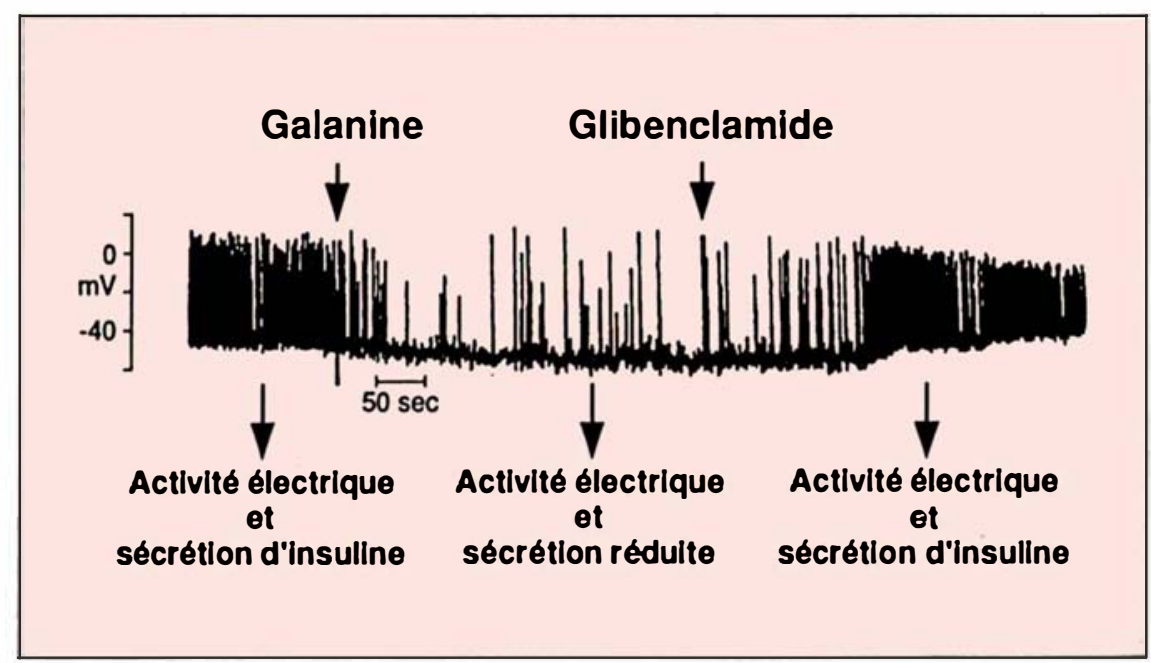

Figure 4. Effet de la galanine sur l'activité électrique de la cellule $\beta$ linsulinome). La cellule est mise en situation "sécrétrice", elle engendre une activité électrique spontanée (concentration élevée d'ATP intracellulaire). La galanine hyperpolarise en ouvrant les canaux $K_{A T P}$. Cette hyperpolarisation diminue considérablement l'activité électrique et par conséquent l'entrée de $\mathrm{Ca}^{2+}$ et la sécrétion. Le glibenclamide rétablit l'une et l'autre.
Les ouvreurs de canaux $\mathrm{K}_{\mathrm{ATP}} \mathrm{du}$ tion, trouveront probablement des affection dans laquelle ils pourront autres types de médicaments disponibles (inhibiteurs calciques, diurétiques, $\beta$-bloquants, inhibiteurs d'enzyme de conversion), mais, plus muscle lisse (inhibiteurs de contrac- 
intéressant encore, dans d'autres anomalies du muscle lisse telles l'asthme ou l'incontinence urinaire, peut-être aussi dans certaines affections du tractus gastro-intestinal.

Les canaux $K_{\text {ATP }}$ cardiaques, leur rôle dans les anomalies d'excitabilité de la cellule ischémiée

C'est dans la cellule cardiaque ventriculaire que les canaux $\mathrm{K}_{\mathrm{ATP}}$ ont été identifiés pour la première fois [10]. Leur rôle fonctionnel dans la cellule cardiaque en situation physiologique normale est encore mystérieux. Il est probable que les canaux $\mathrm{K}_{\mathrm{ATP}}$ cardiaques soient la cible d'hormones ou de neurotransmetteurs qui règlent son activité comme la somatostatine et la galanine le font dans la cellule $\beta$ pancréatique. Le problème qui sera traité ici est celui de l'implication de ces canaux dans l'ischémie cardiaque. Deux événements importants se produisent dans une cellule cardiaque ischémiée : l'acidification cytoplasmique et la diminution intracellulaire d'ATP. La diminution d'ATP cytoplasmique a pour effet d'ouvrir les canaux $\mathrm{K}_{\mathrm{ATP}}$ et par là de raccourcir la durée du potentiel d'action [11-12] (figure 6). Il est bien connu que ce raccourcissement est très largement responsable des anomalies d'électrogenèse observées pendant l'ischémie et qui sont associées aux fibrillations ventriculaires [13].

$\mathrm{Ce}$ raccourcissement $\mathrm{du}$ potentiel d'action dans la cellule ischémiée, dû à l'ouverture des canaux $\mathrm{K}_{\mathrm{ATP}}$, est " annulé " par application des sulfonylurées qui permettent donc clairement d'attribuer la modification du potentiel d'action à ces canaux [12] C'est grâce aux sulfonylurées antidiabétiques que le mécanisme moléculaire des anomalies électriques de la cellule cardiaque ischémiée est désormais connu.

Les canaux $K_{\text {ATP }}$ sensibles aux sulfonylurées sont présents dans le système nerveux central. Rôle physiologique et physiopathologique

C'est la présence de récepteurs de haute affinité aux sulfonylurées antidiabétiques qui a, la première, permis d'identifier la présence de canaux $\mathrm{m} / \mathrm{s} n^{\circ} 3$ vol. 6 , mars 90

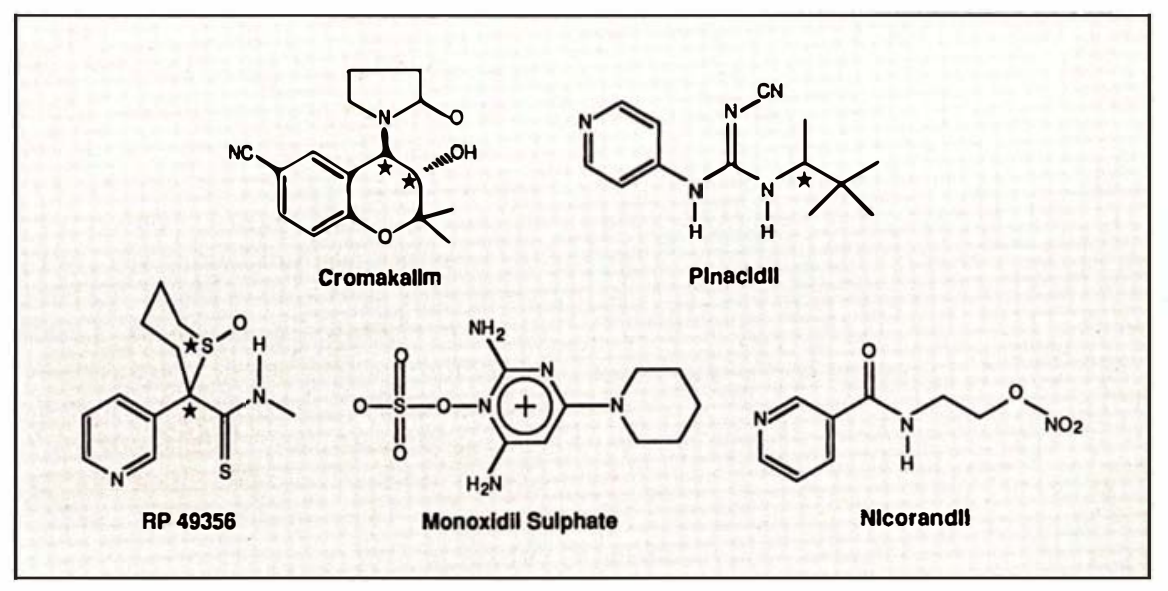

Figure 5. Les différentes classes d'ouvreurs de canaux potassium.

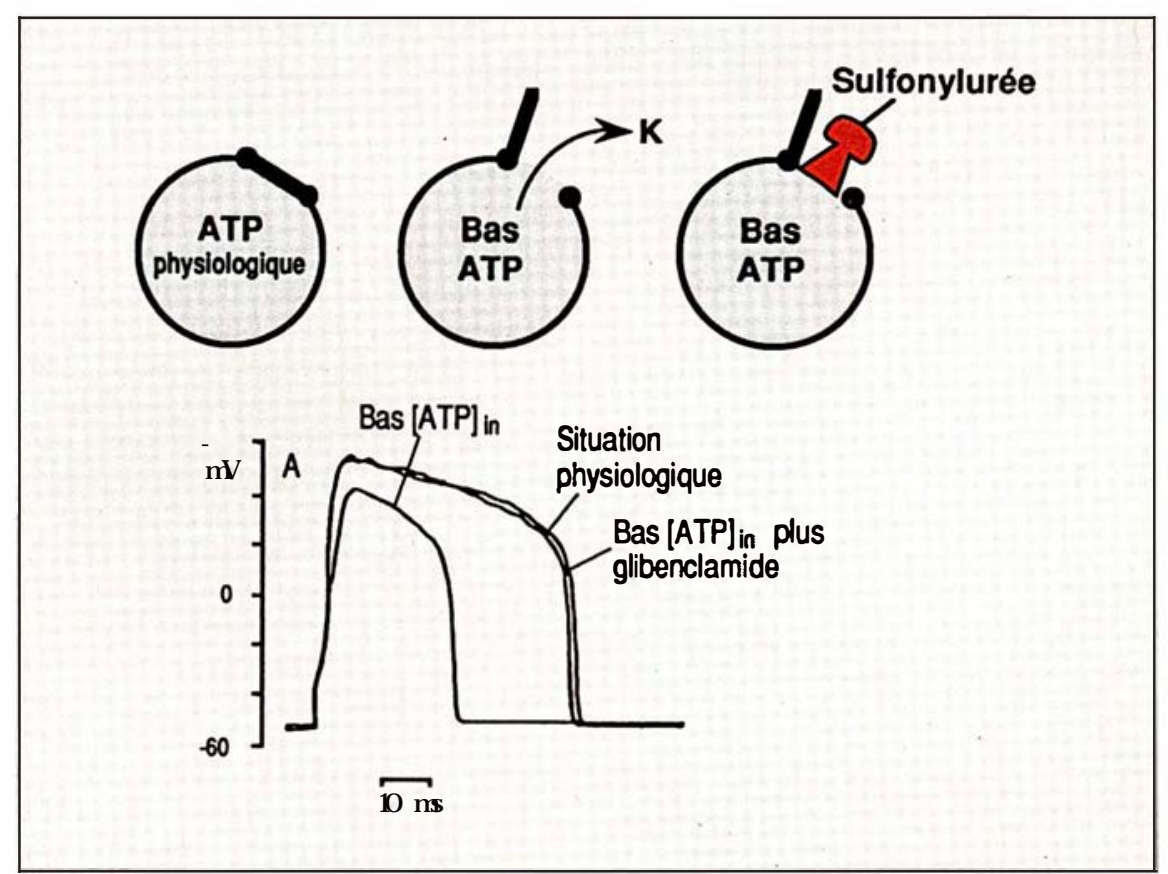

Figure 6. Les canaux $K_{\text {AtP }}$ de la cellule cardiaque sont impliqués dans les perturbations électrogéniques induites par I'ischémie. La diminution de la concentration intracellulaire d'ATP, qui mime l'ischémie, raccourcit le potentiel d'action. Ce raccourcissement est corrigé par le glibenclamide qui bloque le canal $K_{A T P}$.

$\mathrm{K}_{\mathrm{ATP}}$ dans le système nerveux central [5]. L'autoradiographie quantitative de ces récepteurs a permis une localisation très précise de ces canaux [14]. Les densités les plus grandes se trouvent dans la substance noire et le globus pallidus, mais les canaux $\mathrm{K}_{\mathrm{ATP}}$ associés aux récepteurs de sulfonylurées se trouvent dans bien d'autres localisations, et en particulier dans l'hippocampe, siège d'événements primaires de mémorisation et cible privilégiée des destructions cellulaires provoquées par 


\section{RÉFÉRENCES}

12. Fosset M, De Weille JR, Green RD Schmid-Antomarchi H, Lazdunski M. Antidiabetic sulfonylureas control action potential properties in heart cells via high affinity receptors that are linked to ATP-dependent $\mathrm{K}^{+}$channels. J Biol Chem 1988; 263 : 7933-6.

13. Vleugels A, Vereecke J, Carmeliet E. Ionic currents during hypoxia in voltageclamped cat ventricular muscle. Circ Res $1980 ; 47: 501-8$.

14. Mourre C, Ben Ari Y, Bernardi $H$, Forest M, Lazdunski M. Antidiabetic sulfonylureas : localization of binding sites in the brain and effects on the hyperpolarization induced by anoxia in hippocampal slices. Brain Res 1989 ; 486 : 159-64.

15. Siesjö BK. Calcium, ischemia and death of brain cells. Ann NY Acad Sci 1988 ; 522 : 638-61.

16. Amoroso S, Schmid-Antomarchi $\mathrm{H}$ Fosset M, Lazdunski M. Glucose, antidiabetic sulfonylureas and neurotransmitter release. Role of ATP-sensitive $\mathrm{K}^{+}$channels. Science 1990 (sous presse).

17. Cale $\mathrm{K}$. Role of the substantia nigra in GABA-mediated anticonvulsant action. Adv Neurol 1986 ; 44 : 343-64.

18. Hansen AJ. Effect of anoxia on ion distribution in the brain. Physiol Rev 1985 . $65: 101-48$

19. Ben Ari Y, Lazdunski M. Galanin protects hippocampal neurons from the func tional effects of anoxia. Eur J Pharm 1989 ; $165: 331-2$

20. Gandolfo G, Gottesmann C, Bidard JN, Lazdunski $\mathrm{M}$. $\mathrm{K}^{+}$channel opener prevent epilepsy induced by the bee venom peptide MCD. Eur J Pharm 1989; 159 : 329-30.

21. Gandolfo G, Romettino S, Gottesmann $\mathrm{C}$, et al. $\mathrm{K}^{+}$channel openers decrease seizures in genetically epileptic rats. Eur $J$ Pharm $1989 ; 167: 181-3$.

22. Saji M, Reis DJ. Delayed transneuronal death of substantia nigra neurons prevented by $\boldsymbol{\gamma}$-aminobutyric acid agonist. Science 1987 ; $235: 66-9$.

23. Hosey MM, Lazdunski M. Calcium channels : molecular pharmacology, struc ture and regulation. J Membrane Biol 1988 ; 104: 81-105. l'anoxie ou l'ischémie cérébrale [15]. Quel est le rôle physiologique des canaux $\mathrm{K}_{\mathrm{ATP}}$ dans le cerveau ? Sontils associés à la sécrétion de neurotransmetteurs comme ils sont associés à la sécrétion d'insuline dans la cellule $\beta$ pancréatique? La plus grande partie des synapses situées dans la substance noire et le globus pallidus contiennent de l'acide $\boldsymbol{\gamma}$-aminobutyrique (GABA), le plus classique des neurotransmetteurs inhibiteurs. Le GABA inhibe l'activité électrique des neurones dopaminergiques de la substance noire.

L'augmentation de la concentration de glucose extracellulaire stimule la sécrétion synaptique de GABA dans la substance noire [16] comme elle stimulait la sécrétion d'insuline par les îlots de Langherans. L'application des sulfonylurées stimulateurs de la sécrétion d'insuline provoque elle aussi la sécrétion du GABA de la substance noire [16]. On retrouve donc dans le cerveau des mécanismes déjà présents dans le pancréas endocrine. Les canaux $\mathrm{K}_{\text {ATP }}$ sont, cette fois-ci, situés au niveau synaptique ; leur fermeture par le glucose (indirecte) ou les sulfonylurées (directe) entraîne une dépolarisation de la synapse, une entrée de $\mathrm{Ca}^{2+}$ par les canaux $\mathrm{Ca}^{2+}$ synaptiques et une sécrétion de neurotransmetteurs (figure 7). Les canaux $\mathrm{K}_{\mathrm{ATP}}$ du cerveau ont une pharmacologie légèrement différente des canaux $\mathrm{K}_{\mathrm{ATP}}$ du pancréas endocrine et pourraient bien être codés par un ou des gènes distincts. Quelles sont les conséquences physiologiques et physiopathologiques de ces observations? L'innervation GABA-ergique de la substance noire (qui vient du striatum) a une fonction anti-épileptique essentielle [17]. L'inhibition des neurones dopaminergiques par le GABA joue un rôle clé dans l'empêchement de la propagation des crises épileptiques. Une hypoglycémie importante, observée par exemple chez le sujet diabétique, est susceptible d'engendrer un état de convulsions, suivi éventuellement d'un état de coma. Les hypoglycémies provoquées à l'insuline ont d'ailleurs été utilisées dans le passé pour créer des situations d'" électrochocs ". La relation moléculaire entre l'état hypoglycémique et la susceptibilité aux convulsions apparaît désor-

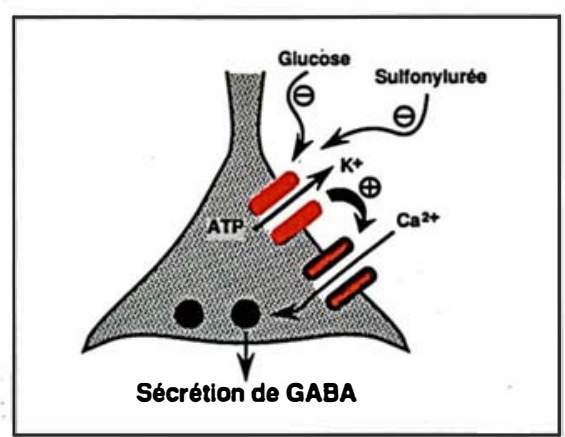

Figure 7. Lactivité sécrétricê des synapses de la substance noire est sensible au glucose et aux sulfonylurées. Le glucose et les sulfonylurées entraînent une fermeture du canal $K_{A T p}$ produisant ainsi une dépolarisation qui active $\oplus$ elle-même les canaux $\mathrm{Ca}^{2+}$, provoque l'entrée de $\mathrm{Ca}^{2+}$ et la sécrétion d'acide $\gamma$ aminobutyrique (GABA).

mais assez clairement [16]. La diminution de la concentration de glucose baignant la partie réticulée de la substance noire où se trouvent les synapses GABA-ergiques diminuera la sécrétion synaptique de GABA, diminuera l'inhibition des neurones dopaminergiques et diminuera considérablement la protection contre les états convulsifs et épileptiques. A l'inverse, un état d'hyperglycémie trop accentué conduira à une inhibition forte de l'activité des neurones dopaminergiques qui explique (ou qui peut expliquer) les modifications d'activité locomotrice observées chez l'animal diabétique rendu hyperglycémique [16] et probablement aussi chez l'homme diabétique.

L'un des événements moléculaires importants de l'ischémie cérébrale, comme de l'ischémie cardiaque, est la diminution de la concentration intracellulaire d'ATP. Cette diminution se produit aussi en cas d'anoxie [16]. Dans tous les cas, cette altération du niveau d'ATP intracellulaire entraînera une ouverture des canaux $\mathrm{K}_{\mathrm{ATP}}$ et une sortie du $\mathrm{K}^{+}$ intracellulaire. C'est probablement là 
l'interprétation d'un phénomène bien connu, mais qui était resté jusqu'ici inexpliqué, l'accumulation de $\mathrm{K}^{+}$ dans l'espace cérébral interstitiel dès qu'un animal a subi un arrêt cardiaque [18]. Cette sortie de $\mathrm{K}^{+}$avec accumulation extracellulaire aura des conséquences dramatiques sur la viabilité des neurones, en particulier dans l'hippocampe, structure particulièrement vulnérable à une atteinte ischémique. L'accumulation de $\mathrm{K}^{+}$ extracellulaire conduira à une chaîne d'événements incluant: (1) une dépolarisation des synapses excitatrices à glutamate qui libèrent leur neurotransmetteur; (2) une activation par le glutamate (transmetteur excitotoxique) de ses récepteurs spécifiques dont le récepteur NMDA, qui est directement couplé un canal perméable au $\mathrm{Ca}^{2+}$; (3) un envahissement massif de $\mathrm{Ca}^{2+}$ qui ne pourra plus ni ressortir de la cellule cible du glutamate, ni être ségrégé dans les dispositifs de stockage calcique intracellulaire faute d'ATP nécessaire au fonctionnement des $\mathrm{Ca}^{2+}$-ATPases qui évacuent le $\mathrm{Ca}^{2+}$ vers l'extérieur ou vers le réticulum endosplasmique ; (4) une activation via cette augmentation de $\mathrm{Ca}^{2+}$ intracellulaire d'une série d'enzymes activées par le $\mathrm{Ca}^{2+}$ (des phospholipases et des protéases) qui détruisent les structures cellulaires, ainsi qu'à une altération $\mathrm{Ca}^{2+}$-dépendante des structures du cytosquelette, tous processus qui entraînent la mort cellulaire.

L'anoxie, comme l'ischémie, libère du glutamate dans l'hippocampe et cette libération est inhibée par la galanine [19] dont on a précédemment vu l'action sur les canaux $\mathrm{K}_{\mathrm{ATP}}$-dépendants dans le pancréas.

\section{Les perspectives}

Les découvertes exposées dans cet article sont susceptibles d'avoir des prolongements dans bien des domaines. On peut citer en vrac: (1) l'application des ouvreurs de canaux potassiques, relaxants vasculaires, en pathologie du système nerveux central. Le cromakalin a une activité bénéfique sur certains types d'épilepsie [20] et a une activité intéressante sur un modèle animal de "petit mal " [21] ; (2) l'implication potentielle des canaux $\mathrm{K}_{\mathrm{ATP}}$ dans la régu- lation de l'appétit pour les sucres. L'activité électrique de neurones de l'hypothalamus ventro-médian est contrôlée par les composés sucrés. Il est possible que l'activité électrique de cette région du cerveau impliquée dans la régulation de l'appétit soit réglée d'une façon semblable à ce qui a été décrit pour la cellule $\beta$ pancréatique ; (3) il est possible que l'activité des canaux $\mathrm{K}_{\mathrm{ATP}}$-dépendants soit indirectement liée à des processus de dégénérescence neuronale. Nous avons vu plus haut comment ces canaux réglaient la sécrétion du GABA au niveau de la substance noire. La fonctionnalité de l'innervation GABAergique semble être importante pour la survie des neurones dopaminergiques [22].

L'histoire moléculaire, cellulaire et physiopathologique du canal $\mathrm{K}_{\mathrm{ATP}}$ n'aurait pu être écrite sans la découverte que ces canaux étaient la cible des sulf onylurées susceptibles de se lier à lui avec une très haute affinité. On se retrouve ici dans une situation semblable à celle qui vient d'être vécue pour les canaux calciques [23], dont l'étude n'aurait pas été possible sans une pharmacologie de haute affinité, représentée dans ce cas par les différents types d'inhibiteurs calciques, utilisés comme on le sait dans une variété de maladies, et principalement dans le domaine cardiovasculaire.

Il est probable que le clonage moléculaire des ADN codant pour les différents types de canaux $K_{A T P}$ ouvrira des voies nouvelles tant sur le plan cellulaire que sur le plan physiopathologique

\section{Remerciements}

Ces travaux ont bénéficié du soutien financier du Centre national de la recherche scientifique, de l'Institut national de la santé et de la recherche médicale (contrat $n^{\circ} 88.2007$ ) et du MRT. L'auteur remercie les Drs M. Fosset, H. Schmid-Antomarchi, Jan De Weille, S. Amoroso, C. Mourre, H. Bernardi ainsi que Mme I. De Angelis et Mr F. Aguila.

\section{Summary}

ATP dependent $\mathrm{K}^{+}$-channels : unexpected consequences of investigations on sulf onylureas

Sulfonylureas are "old" drugs for diabetes of type 2. They now rank as essential tools for the analysis of the function, regulation and physiopathological implications of one very new class of ionic channels, that of $\mathrm{K}+$ channels which are sensitive to variations of intracellular ATP concentrations. The channels have an essential role in insulin secretion. They are also present with an important physiological function in cardiac cells, in smooth muscle and in the central nervous system. They are in close connection with a variety of pathologies which rank from diabetes to disorders linked to cerebral ischemia and which include hypertension or cardiac arythmias.

\section{TIRÉS A PART}

M. Lazdunski. 